\title{
COVID-19
}

\section{Health Evidence Summary No.116}

\section{Kerry Millington}

Liverpool School of Tropical Medicine (LSTM)

15 March 2021

This weekly COVID-19 health evidence summary (HES) is based on 3.5 hours of desk-based research. The summary is not intended to be a comprehensive summary of available evidence on COVID-19 but aims to make original documents easily accessible to decision makers which, if relevant to them, they should go to before making decisions.

\section{Clinical characteristics and management}

\begin{tabular}{|c|c|c|c|c|}
\hline $\begin{array}{l}\text { Publication } \\
\text { date }\end{array}$ & Title/URL & $\begin{array}{l}\text { Journal/Article } \\
\text { type }\end{array}$ & Summary & Keywords \\
\hline 12.03.2021 & $\begin{array}{l}\text { Association of } \\
\text { acute } \\
\text { symptoms of } \\
\text { COVID-19 } \\
\text { and } \\
\text { symptoms of } \\
\text { depression in } \\
\text { adults }\end{array}$ & $\begin{array}{l}\text { JAMA I } \\
\text { Research } \\
\text { Letter }\end{array}$ & $\begin{array}{l}\text { - Survey of } 3,904 \\
\text { COVID-19 survivors } \\
\text { between May } 2020 \\
\text { and January } 2021 \text { in } \\
\text { the US found that } \\
52.4 \% \text { met the criteria } \\
\text { for moderate or } \\
\text { greater symptoms of } \\
\text { major depression } \\
\text { These symptoms were } \\
\text { more likely among } \\
\text { younger compared } \\
\text { with older } \\
\text { respondents; among } \\
\text { men compared with } \\
\text { women and among } \\
\text { those with more } \\
\text { severe COVID-19 } \\
\text { illness compared with } \\
\text { lower severity } \\
\text { Authors highlight the } \\
\text { importance of } \\
\text { considering strategies } \\
\text { that might mitigate the } \\
\text { elevated risk of } \\
\text { depressive symptoms } \\
\text { following acute } \\
\text { infection }\end{array}$ & $\begin{array}{l}\text { COVID-19, } \\
\text { depression }\end{array}$ \\
\hline
\end{tabular}




\begin{tabular}{|c|c|c|c|c|}
\hline 11.03 .2021 & $\begin{array}{l}\text { COVID-19 } \\
\text { and its } \\
\text { cardiovascular } \\
\text { effects: a } \\
\text { systematic } \\
\text { review of } \\
\text { prevalence } \\
\text { studies }\end{array}$ & $\begin{array}{l}\text { Cochrane | } \\
\text { Systematic } \\
\text { Review }\end{array}$ & $\begin{array}{l}\text { People who have } \\
\text { diabetes, high blood } \\
\text { pressure or pre- } \\
\text { existing heart } \\
\text { problems are at } \\
\text { greater risk of } \\
\text { developing } \\
\text { complications if they } \\
\text { get COVID-19 } \\
\text { This systematic review } \\
\text { wanted to find out, in } \\
\text { cases of confirmed or } \\
\text { suspected COVID-19, } \\
\text { what are the most } \\
\text { common pre-existing } \\
\text { heart and blood vessel } \\
\text { problems and what } \\
\text { are the most common } \\
\text { complications affected } \\
\text { the heart and blood } \\
\text { vessels in different } \\
\text { settings } \\
\text { Cardiometabolic } \\
\text { comorbidities are } \\
\text { common in people } \\
\text { who are hospitalised } \\
\text { with a COVID-19 } \\
\text { infection, and } \\
\text { cardiovascular } \\
\text { complications are } \\
\text { frequent } \\
\text { The studies were very } \\
\text { different from each } \\
\text { other, did not always } \\
\text { reports results in the } \\
\text { same way or use the } \\
\text { most reliable methods } \\
\text { This review will be } \\
\text { updated with a formal } \\
\text { meta-analysis of } \\
\text { outcomes based on a } \\
\text { more homogeneous } \\
\text { selected subsample of } \\
\text { high-certainty studies } \\
\end{array}$ & $\begin{array}{l}\text { COVID-19, } \\
\text { cardiovascular }\end{array}$ \\
\hline 10.03.2021 & $\begin{array}{l}\text { What is the } \\
\text { recovery rate } \\
\text { and risk of } \\
\text { long-term } \\
\text { consequences } \\
\text { following a } \\
\text { diagnosis of } \\
\text { COVID-19? A } \\
\text { harmonised, }\end{array}$ & $\begin{array}{l}\text { BMJ Open | } \\
\text { Protocol }\end{array}$ & $\begin{array}{l}\text { The assessment of } \\
\text { risk factors for longer } \\
\text { term consequences of } \\
\text { COVID-19 requires a } \\
\text { longitudinal study } \\
\text { linked to data on pre- } \\
\text { existing conditions } \\
\text { and care received } \\
\text { during the acute } \\
\text { phase of illness }\end{array}$ & $\begin{array}{l}\text { Long-term } \\
\text { COVID-19, } \\
\text { protocol }\end{array}$ \\
\hline
\end{tabular}




\begin{tabular}{|c|c|}
\hline $\begin{array}{l}\text { global } \\
\text { longitudinal } \\
\text { observational } \\
\text { study protocol }\end{array}$ & $\begin{array}{l}\text { An international open } \\
\text { access prospective, } \\
\text { observational protocol } \\
\text { to characterise } \\
\text { physical and } \\
\text { psychological } \\
\text { sequelae in patients } \\
\text { post-COVID-19 } \\
\text { hospital discharge } \\
\text { The open access } \\
\text { follow-up survey can } \\
\text { be used by other } \\
\text { follow-up studies to } \\
\text { facilitate standardised, } \\
\text { multisite data } \\
\text { collection to forward } \\
\text { knowledge into long- } \\
\text { term consequences of } \\
\text { cOVID-19 }\end{array}$ \\
\hline
\end{tabular}

\section{Epidemiology and modelling}

\begin{tabular}{|c|c|c|c|c|}
\hline $\begin{array}{l}\text { Publication } \\
\text { date }\end{array}$ & Title/URL & $\begin{array}{l}\text { Journal/Article } \\
\text { type }\end{array}$ & Summary & Keywords \\
\hline 10.03.2021 & $\begin{array}{l}\text { Risk of } \\
\text { mortality in } \\
\text { patients } \\
\text { infected } \\
\text { with } \\
\text { SARS- } \\
\text { CoV-2 } \\
\text { variant of } \\
\text { concern } \\
202012 / 1 \text { : } \\
\text { matched } \\
\text { cohort } \\
\text { study }\end{array}$ & $\begin{array}{l}\text { BMJ | } \\
\text { Research }\end{array}$ & $\begin{array}{l}\text { The SARS-CoV-2 VOC- } \\
202012 / 1 \text {, first detected in } \\
\text { SE England in autumn } 2020 \\
\text { and now spread to } 100+ \\
\text { countries, is more } \\
\text { transmissible than } \\
\text { previously circulating } \\
\text { variants and is known to } \\
\text { increase mortality but before } \\
\text { this study, unbiased } \\
\text { estimates of the mortality } \\
\text { were not available } \\
\text { Cohort study of } 54,906 \\
\text { matched pairs of participants } \\
\text { who tested positive for } \\
\text { SARS-CoV-2 in community } \\
\text { testing centres in UK } \\
\text { between } 1 \text { October } 2020 \\
\text { and } 29 \text { January } 2021, \\
\text { followed-up until } 12 \\
\text { February } 2021 \\
\text { Individuals infected with } \\
\text { SARS-CoV-2 VOC- } \\
202012 / 1, \text { were between } \\
32 \% \text { to } 104 \% \text { (central } \\
\text { estimate } 64 \% \text { more likely to } \\
\text { die than matched individuals }\end{array}$ & $\begin{array}{l}\text { UK variant, } \\
\text { mortality }\end{array}$ \\
\hline
\end{tabular}




\begin{tabular}{|c|c|c|c|c|}
\hline & & & $\begin{array}{l}\text { with previously circulating } \\
\text { SARS-CoV-2 variants } \\
\text { - Absolute risk of death in this } \\
\text { largely unvaccinated } \\
\text { population remains low, but } \\
\text { clinicians and public health } \\
\text { officials should be aware } \\
\text { that a higher mortality rate is } \\
\text { likely if the response } \\
\text { remains unchanged }\end{array}$ & \\
\hline 09.03.2021 & $\begin{array}{l}\text { Prevalence } \\
\text { of SARS- } \\
\text { CoV-2 in } \\
\text { six districts } \\
\text { in Zambia } \\
\text { in July, } \\
2020 \text { : a } \\
\text { cross- } \\
\text { sectional } \\
\text { cluster } \\
\text { sample } \\
\text { survey }\end{array}$ & $\begin{array}{l}\text { The Lancet } \\
\text { Global Health } \\
\text { | Article }\end{array}$ & $\begin{array}{l}\text { Number of SARS-CoV-2 } \\
\text { infections in Zambia are } \\
\text { likely to be higher than } \\
\text { confirmed cases counts } \\
\text { (20,000 laboratory- } \\
\text { confirmed cases between } \\
\text { March and December 2020) } \\
\text { because many infected have } \\
\text { mild or no symptoms and } \\
\text { due to limited testing } \\
\text { capacity and surveillance } \\
\text { systems } \\
\text { Here, a population-based } \\
\text { household survey was used } \\
\text { to estimate SARS-CoV-2 } \\
\text { prevalence in six districts of } \\
\text { Zambia in July } 2020 \\
4258 \text { people from } 1866 \\
\text { households participated } \\
\text { An estimated } 454,708 \\
\text { SARS-CoV-2 infections } \\
\text { (95\% Cl 312,705 to } \\
596,713) \text { occurred in the six } \\
\text { districts between March and } \\
\text { July 2020, much higher } \\
\text { when compared with } 4917 \\
\text { laboratory-confirmed cases } \\
\text { reported in official statistics } \\
\text { from the Zambia National } \\
\text { Public Health Institute }\end{array}$ & $\begin{array}{l}\text { SARS- } \\
\text { CoV-2 } \\
\text { prevalence, } \\
\text { population- } \\
\text { based } \\
\text { household } \\
\text { survey, } \\
\text { Zambia }\end{array}$ \\
\hline 08.03.2021 & $\begin{array}{l}\text { Serological } \\
\text { evidence } \\
\text { of human } \\
\text { infection } \\
\text { with } \\
\text { SARS- } \\
\text { CoV-2: a } \\
\text { systematic } \\
\text { review and } \\
\text { meta- } \\
\text { analysis }\end{array}$ & $\begin{array}{l}\text { The Lancet } \\
\text { Global Health } \\
\text { | Article }\end{array}$ & $\begin{array}{l}\text { Meta-analysis of } 404 \\
\text { published and preprint } \\
\text { serological studies from } \\
\text { across the globe } \\
\text { A higher prevalence of } \\
\text { SARS-CoV-2-specific } \\
\text { antibodies was observed in } \\
\text { close contacts (18.0\%) and } \\
\text { high-risk health care workers } \\
\text { (17.1\%) than in low-risk } \\
\text { health care workers (4.3\%) } \\
\text { and the general population } \\
\text { (8.0\%) - far from herd } \\
\text { immunity }\end{array}$ & serology \\
\hline
\end{tabular}




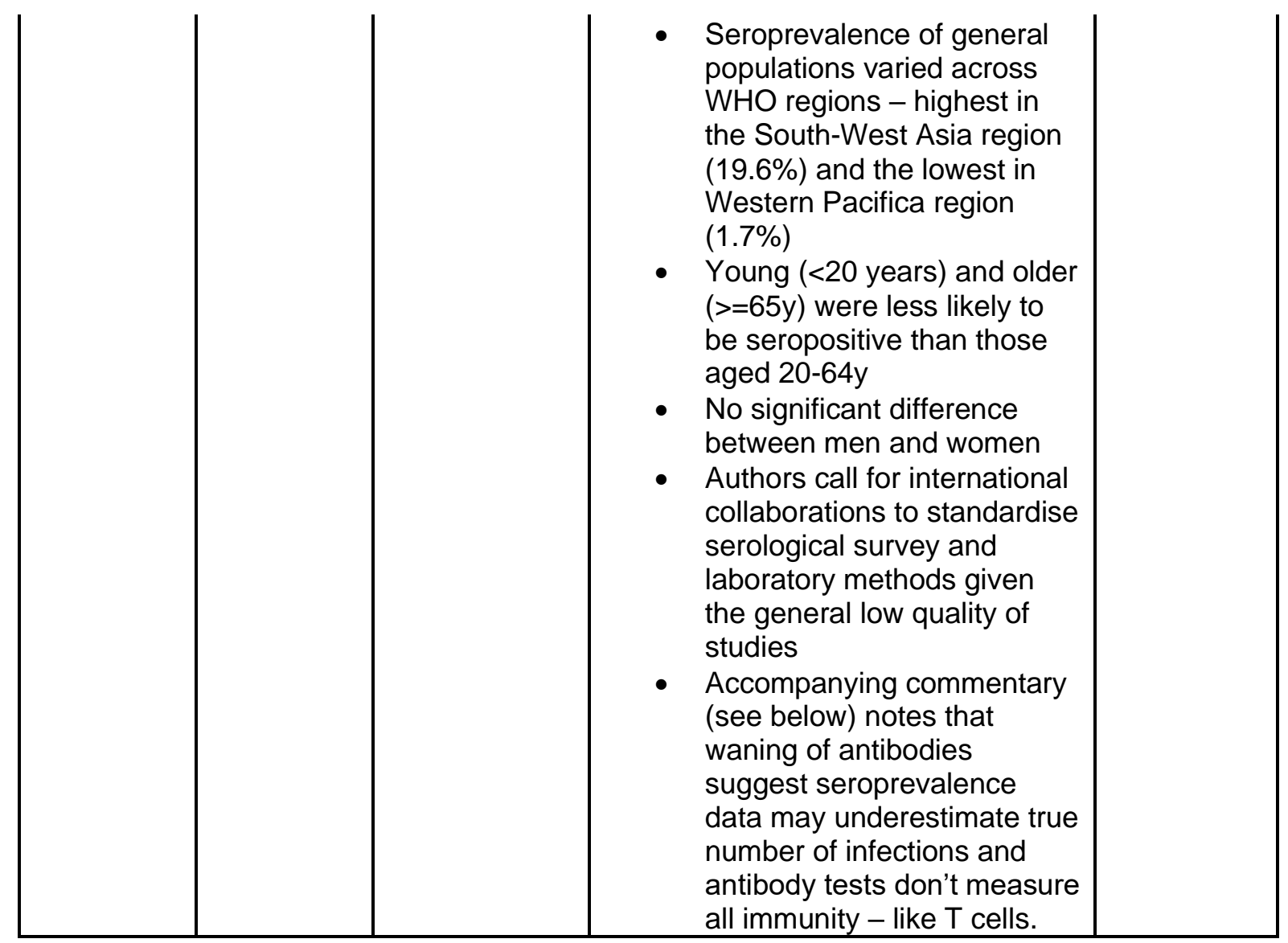

\section{Therapeutics}

\begin{tabular}{|c|c|c|c|c|}
\hline $\begin{array}{l}\text { Publication } \\
\text { date }\end{array}$ & Title/URL & $\begin{array}{l}\text { Journal/Article } \\
\text { type }\end{array}$ & Summary & Keywords \\
\hline 10.03 .2021 & $\begin{array}{l}\text { Convalescent } \\
\text { plasma in } \\
\text { patients } \\
\text { admitted to } \\
\text { hospital with } \\
\text { COVID-19 } \\
\text { (RECOVERY): } \\
\text { a randomised, } \\
\text { controlled, } \\
\text { open-label } \\
\text { platform trial }\end{array}$ & $\begin{array}{l}\text { medRxiv | } \\
\text { preprint (not } \\
\text { peer } \\
\text { reviewed) }\end{array}$ & 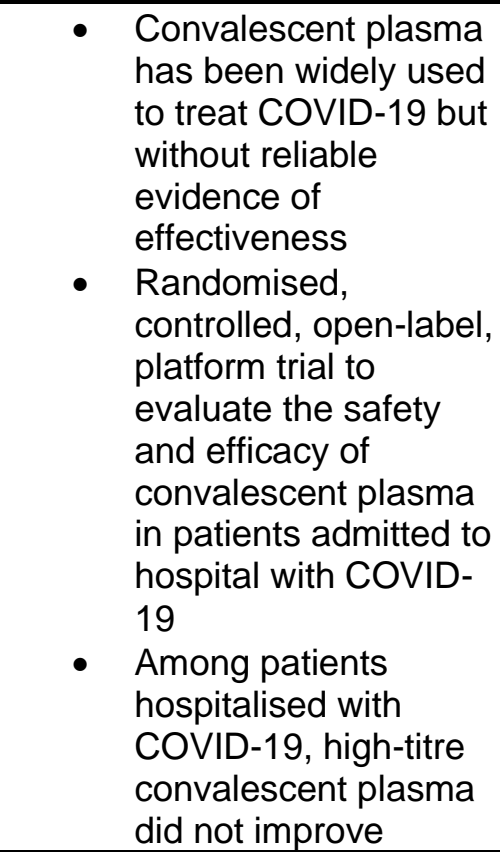 & $\begin{array}{l}\text { Convalescent } \\
\text { plasma }\end{array}$ \\
\hline
\end{tabular}




\begin{tabular}{|l|l|l|} 
& & $\begin{array}{l}\text { survival at 28-days or } \\
\text { other prespecified } \\
\text { clinical outcomes }\end{array}$ \\
\hline
\end{tabular}

\section{Vaccines}

\begin{tabular}{|c|c|c|c|c|}
\hline $\begin{array}{l}\text { Publication } \\
\text { date }\end{array}$ & Title/URL & $\begin{array}{l}\text { Journal/Article } \\
\text { type }\end{array}$ & Summary & Keywords \\
\hline 11.03 .2021 & $\begin{array}{l}\text { ChAdOx1 } \\
\text { nCoV-19 } \\
\text { (AZD1222) } \\
\text { protects against } \\
\text { SARS-CoV-2 } \\
\text { B.1.351 and } \\
\text { B.1.1.7 }\end{array}$ & $\begin{array}{l}\text { bioRxiv | } \\
\text { preprint (not } \\
\text { peer } \\
\text { reviewed) }\end{array}$ & $\begin{array}{l}\text { - Investigation of } \\
\text { ChAdOx1 nCoV-19 } \\
\text { (AZD1222) vaccine } \\
\text { efficacy against } \\
\text { SARS-CoV-2 VoC } \\
\text { B.1.1.7 and B.1.351 } \\
\text { in Syrian hamsters, in } \\
\text { which protection } \\
\text { against SARS-CoV-2 } \\
\text { disease and } \\
\text { pneumonia in } \\
\text { hamsters vaccinated } \\
\text { with a single dose of } \\
\text { ChAdOx1 nCoV-19 } \\
\text { has been shown } \\
\text { Lack of disease in } \\
\text { hamsters vaccinated } \\
\text { with ChAdOx1 nCoV- } \\
19 \text { when infected with } \\
\text { B1.1.7 or B.1.351 } \\
\text { demonstrate the } \\
\text { effectiveness of the } \\
\text { ChAdOx1 nCoV-19 } \\
\text { vaccine against } \\
\text { clinical disease } \\
\text { caused by these } \\
\text { variants }\end{array}$ & $\begin{array}{l}\text { Oxford- } \\
\text { AstraZeneca } \\
\text { vaccine, } \\
\text { efficacy, UK } \\
\text { variant, } \\
\text { South } \\
\text { African } \\
\text { variant, } \\
\text { hamsters }\end{array}$ \\
\hline 09.03.2021 & $\begin{array}{l}\text { Epidemiological } \\
\text { and } \\
\text { evolutionary } \\
\text { considerations } \\
\text { of SARS-CoV-2 } \\
\text { vaccine dosing } \\
\text { regimes }\end{array}$ & $\begin{array}{l}\text { Science | } \\
\text { Research } \\
\text { Article }\end{array}$ & $\begin{array}{l}\text { Various vaccine } \\
\text { deployment strategies } \\
\text { are being proposed } \\
\text { due to vaccine dose } \\
\text { shortages and } \\
\text { logistical challenges } \\
\text { Two critical issues: } \\
\text { how will timing of } \\
\text { delivery of the second } \\
\text { does affect immunity } \\
\text { and evolution of viral } \\
\text { immune escape from } \\
\text { a build-up of partially } \\
\text { immune individuals } \\
\text { - Here, building on an } \\
\text { existing immune- } \\
\text { epidemiological }\end{array}$ & $\begin{array}{l}\text { Vaccine } \\
\text { deployment } \\
\text { strategies }\end{array}$ \\
\hline
\end{tabular}




\begin{tabular}{|c|c|c|c|c|}
\hline & & & $\begin{array}{l}\text { model, short-term } \\
\text { outcome is that one } \\
\text { dose generally } \\
\text { decreases infections } \\
\text { but longer-term } \\
\text { outcomes depend on } \\
\text { relative immune } \\
\text { robustness } \\
\text { Exploring three } \\
\text { scenarios, a one-dose } \\
\text { policy may increase } \\
\text { the potential for } \\
\text { antigenic evolution } \\
\text { under certain } \\
\text { conditions of partial } \\
\text { population immunity }\end{array}$ & \\
\hline 08.03.2021 & $\begin{array}{l}\text { Safety and } \\
\text { immunogenicity } \\
\text { of an } \\
\text { inactivated } \\
\text { SARS-CoV-2 } \\
\text { vaccine, } \\
\text { BBV152: } \\
\text { interim results } \\
\text { from a double- } \\
\text { blind, } \\
\text { randomised, } \\
\text { multicentre, } \\
\text { phase } 2 \text { trial, } \\
\text { and 3-month } \\
\text { follow-up of a } \\
\text { double-blind, } \\
\text { randomised } \\
\text { phase } 1 \text { trial }\end{array}$ & $\begin{array}{l}\text { The Lancet } \\
\text { Infectious } \\
\text { Diseases | } \\
\text { Article }\end{array}$ & $\begin{array}{l}\text { Report on interim } \\
\text { findings of the phase } \\
2 \text { trial on the } \\
\text { immunogenicity and } \\
\text { safety of BBV152, a } \\
\text { whole-virion } \\
\text { inactivated SARS- } \\
\text { CoV-2 vaccine, with } \\
\text { first dose } \\
\text { administered on day } 0 \\
\text { and the second dose } \\
\text { on day } 28 \\
\text { In the phase } 1 \text { trial, } \\
\text { BBV152 induced high } \\
\text { neutralising antibody } \\
\text { responses which } \\
\text { remained elevated in } \\
\text { all participants } 3 \\
\text { months after the } \\
\text { second dose } \\
\text { In the phase } 2 \text { trial, } \\
\text { BB152 showed better } \\
\text { reactogenicity and } \\
\text { safety outcomes and } \\
\text { enhanced immunity } \\
\text { compared with the } \\
\text { phase } 1 \text { trial } \\
\text { This vaccine } \\
\text { candidate (6ug with } \\
\text { Algel-IMDG } \\
\text { formulation) is now in } \\
\text { a phase } 3 \text { efficacy } \\
\text { trial } \\
\end{array}$ & $\begin{array}{l}\text { BBV152 } \\
\text { vaccine } \\
\text { candidate }\end{array}$ \\
\hline
\end{tabular}




\section{Indirect impact of COVID-19}

\begin{tabular}{|c|c|c|c|c|}
\hline $\begin{array}{l}\text { Publication } \\
\text { date }\end{array}$ & Title/URL & $\begin{array}{l}\text { Journal/Article } \\
\text { type }\end{array}$ & Summary & Keywords \\
\hline 14.03 .2021 & $\begin{array}{l}\text { Small and } \\
\text { sick newborn } \\
\text { care during } \\
\text { the COVID- } \\
19 \\
\text { pandemic: } \\
\text { global survey } \\
\text { and thematic } \\
\text { analysis of } \\
\text { healthcare } \\
\text { providers' } \\
\text { voices and } \\
\text { experiences }\end{array}$ & $\begin{array}{l}\text { BMJ Global } \\
\text { Health | } \\
\text { Original } \\
\text { research }\end{array}$ & $\begin{array}{l}\text { - Disruptions to maternity } \\
\text { care caused by the } \\
\text { COVID-19 pandemic have } \\
\text { been surveyed but not } \\
\text { those related to vulnerable } \\
\text { small newborns } \\
\text { - Using a widely } \\
\text { disseminated online survey } \\
\text { in three languages, this } \\
\text { study reached out to } \\
\text { neonatal healthcare } \\
\text { providers worldwide, } \\
\text { undertook thematic } \\
\text { analysis of healthcare } \\
\text { providers' experiences and } \\
\text { proposed mitigation } \\
\text { strategies } \\
\text { Analysis of } 1120 \\
\text { responses from } 62 \\
\text { countries, mainly LMICs } \\
\text { Newborn care providers } \\
\text { are stressed and } \\
\text { compromised and there is } \\
\text { a lack of clarity and } \\
\text { guidelines regarding care } \\
\text { of small newborns during } \\
\text { the pandemic } \\
\text { Life-saving interventions, } \\
\text { such as KMC, must be } \\
\text { urgently protected } \\
\end{array}$ & $\begin{array}{l}\text { Newborn } \\
\text { care }\end{array}$ \\
\hline 09.03 .2021 & $\begin{array}{l}\text { Violence } \\
\text { Against } \\
\text { Women } \\
\text { Prevalence } \\
\text { Estimates, } \\
2018\end{array}$ & WHO | Report & $\begin{array}{l}\text { Data from the largest study } \\
\text { to-date, conducted by } \\
\text { WHO on behalf of a } \\
\text { special working group of } \\
\text { the UN, of the prevalence } \\
\text { of violence against women } \\
\text { - Based on data from } 2000 \\
\text { to } 2018 \text { - a update on } \\
\text { previous estimates } \\
\text { released in } 2013 \\
\text { - Numbers though do not } \\
\text { reflect ongoing impact of } \\
\text { the COVID-19 pandemic } \\
\text { which has further } \\
\text { increased women's } \\
\text { exposure to violence due } \\
\text { to non-pharmaceutical } \\
\text { interventions such as }\end{array}$ & $\begin{array}{l}\text { Violence } \\
\text { against } \\
\text { women }\end{array}$ \\
\hline
\end{tabular}




\section{Health systems}

\begin{tabular}{|c|c|c|c|c|}
\hline $\begin{array}{l}\text { Publication } \\
\text { date }\end{array}$ & Title/URL & $\begin{array}{l}\text { Journal/Article } \\
\text { type }\end{array}$ & Summary & Keywords \\
\hline 10.03 .2021 & $\begin{array}{l}\text { On the } \\
\text { importance of } \\
\text { primary and } \\
\text { community } \\
\text { healthcare in } \\
\text { relation to } \\
\text { global health } \\
\text { and } \\
\text { environmental } \\
\text { threats: } \\
\text { lessons from } \\
\text { the COVID-19 } \\
\text { crisis }\end{array}$ & $\begin{array}{l}\text { BMJ Global } \\
\text { Health | } \\
\text { Analysis }\end{array}$ & $\begin{array}{l}\text { Analysis of the role and } \\
\text { weaknesses of primary } \\
\text { and community } \\
\text { healthcare worldwide } \\
\text { This paper proposes that } \\
\text { primary and community } \\
\text { healthcare should (i) } \\
\text { support local problem- } \\
\text { solving efforts and serve } \\
\text { as a partner in } \\
\text { innovative approaches } \\
\text { to safeguarding } \\
\text { community well-being; } \\
\text { and (ii) understand the } \\
\text { local environment and } \\
\text { health risks in the } \\
\text { context of the global } \\
\text { health perspective }\end{array}$ & $\begin{array}{l}\text { Primary } \\
\text { and } \\
\text { community } \\
\text { healthcare }\end{array}$ \\
\hline
\end{tabular}

\section{Comments, Editorials, Opinions, Blogs, News}

\begin{tabular}{|l|l|l|}
\hline $\begin{array}{l}\text { Publication } \\
\text { date }\end{array}$ & Title/URL & Journal | Article type \\
\hline 15.03.2021 & $\begin{array}{l}\text { German, France and Italy suspend Oxford Covid } \\
\text { vaccine }\end{array}$ & The Guardian | News \\
\hline March 2021 & $\begin{array}{l}\text { Measles: the long walk to elimination drawn out by } \\
\text { COVID-19 }\end{array}$ & $\begin{array}{l}\text { The Lancet Global Health } \\
\text { I Comment }\end{array}$ \\
\hline 15.03 .2021 & $\begin{array}{l}\text { Regular booster vaccines are the future in battle with } \\
\text { COVID-19 virus, expert says }\end{array}$ & Reuters | News \\
\hline 12.03.2021 & $\begin{array}{l}\text { Ghana's COVID-19 response: the Black Star can do } \\
\text { even better }\end{array}$ & $\begin{array}{l}\text { BMJ Global Health } \\
\text { Commentary }\end{array}$ \\
\hline
\end{tabular}




\begin{tabular}{|c|c|c|}
\hline 12.03.2021 & $\begin{array}{l}\text { COVID antibody treatments show promise for } \\
\text { preventing severe disease }\end{array}$ & Nature | News \\
\hline 12.03.2021 & $\begin{array}{l}\text { Long Covid more likely in working-age women than } \\
\text { in men - study }\end{array}$ & The Guardian | News \\
\hline 11.03 .2021 & $\begin{array}{l}\text { Is Covid-19 reshaping our approach to development } \\
\text { research? }\end{array}$ & $\begin{array}{l}\text { IDS | Opinion (Peter } \\
\text { Taylor) }\end{array}$ \\
\hline 11.03 .2021 & $\begin{array}{l}\text { COVID-19 cases in Africa to top } 4 \text { million, vaccine } \\
\text { rollout underway }\end{array}$ & WHO Africa | News \\
\hline 11.03 .2021 & $\begin{array}{l}\text { Redressing the impact of COVID-19 on medical } \\
\text { education in Africa: the need for collective action }\end{array}$ & $\begin{array}{l}\text { BMJ Global Health } \\
\text { Commentary }\end{array}$ \\
\hline 10.03.2021 & $\begin{array}{l}\text { Deadly pig disease could have led to Covid spillover } \\
\text { to humans, analysis suggests }\end{array}$ & The Guardian | News \\
\hline 09.03 .2021 & $\begin{array}{l}\text { Mitigating gender-based violence risk in the context } \\
\text { of COVID-19: lessons from humanitarian crises }\end{array}$ & $\begin{array}{l}\text { BMJ Global Health } \\
\text { Commentary }\end{array}$ \\
\hline 09.03 .2021 & $\begin{array}{l}3 \text { medical innovations fueled by COVID-19 that will } \\
\text { outlast the pandemic }\end{array}$ & The Conversation \\
\hline 09.03 .2021 & $\begin{array}{l}\text { Understanding the impact of COVID-19 on the } \\
\text { private health sector in Africa }\end{array}$ & BMJ Global Health | Blog \\
\hline 08.03 .2021 & $\begin{array}{l}\text { COVID-19 serosurveys for public health decision } \\
\text { making }\end{array}$ & $\begin{array}{l}\text { The Lancet Global Health } \\
\text { | Commentary }\end{array}$ \\
\hline 08.03.2021 & A moral failure in pandemic response & CGD | Blog \\
\hline 08.03 .2021 & $\begin{array}{l}\text { Financing for Global Health Security and pandemic } \\
\text { preparedness: taking stock and what's next }\end{array}$ & CGD | Blog \\
\hline 05.03 .2021 & Overcoming the gender gap in Covid experience & IDS | News \\
\hline 04.03 .2021 & Covid-19: A stress test for trust? & IDS | Briefing \\
\hline
\end{tabular}




\section{Dashboards \& Trackers}

\begin{tabular}{|c|c|c|c|c|c|c|c|}
\hline $\begin{array}{l}\text { Cases \& deaths: } \\
\text { Global }\end{array}$ & $\begin{array}{l}\text { Cases \& } \\
\text { deaths: } \\
\text { Regional }\end{array}$ & $\begin{array}{l}\text { Cases \& } \quad \& \\
\text { deaths: } \\
\text { Country }\end{array}$ & $\begin{array}{l}\text { Living evidence \& } \\
\text { policy maps }\end{array}$ & $\begin{array}{l}\text { Current research } \\
\text { including trials }\end{array}$ & Diagnostics & Treatments & Vaccines \\
\hline WHO sitreps & WHO Africa & Ghana & COVID-NMA & WHO & $\begin{array}{l}\text { FIND SARS- } \\
\text { CoV-2 Test } \\
\text { Tracker }\end{array}$ & $\begin{array}{l}\text { Global COVID- } \\
19 \text { Clinical Trial } \\
\text { Tracker }\end{array}$ & CEPI \\
\hline WHO dashboard & $\begin{array}{l}\text { African } \\
\text { Arguments }\end{array}$ & Indonesia & EPPI Centre & $\begin{array}{l}\text { WHO International } \\
\text { Clinical Trials } \\
\text { Registry Platform } \\
\text { (ICTRP) }\end{array}$ & $\begin{array}{l}\text { FIND SARS- } \\
\text { CoV-2 } \\
\text { Diagnostics: } \\
\text { performance } \\
\text { data }\end{array}$ & $\begin{array}{l}\text { US NIH } \\
\text { registered } \\
\text { clinical trials }\end{array}$ & $\begin{array}{l}\text { Vaccine Centre } \\
\text { LSHTM }\end{array}$ \\
\hline $\begin{array}{l}\text { Johns Hopkins } \\
\text { University }\end{array}$ & $\begin{array}{l}\text { European } \\
\text { CDC }\end{array}$ & Nigeria CDC & $\begin{array}{l}\text { Norwegian } \\
\text { Institute of Public } \\
\text { Health }\end{array}$ & Cytel & $\begin{array}{l}\text { Serology-based } \\
\text { tests for COVID- } \\
19\end{array}$ & Solidarity trial & $\begin{array}{l}\text { COVID-19 } \\
\text { Oxford Vaccine } \\
\text { Trial }\end{array}$ \\
\hline WEF & & Sierra Leone & $\begin{array}{l}\text { Oxford C19 } \\
\text { Government } \\
\text { Response } \\
\text { Tracker } \\
\text { (OxCGRT) }\end{array}$ & US NIH & $\begin{array}{l}\text { Our World in } \\
\text { Data: C19 } \\
\text { Testing }\end{array}$ & $\begin{array}{l}\text { COVID-19 } \\
\text { Therapeutics } \\
\text { Accelerator }\end{array}$ & $\begin{array}{l}\text { COVID-19 } \\
\text { Vaccine Tracker }\end{array}$ \\
\hline
\end{tabular}




\begin{tabular}{|c|c|c|c|c|}
\hline $\begin{array}{l}\text { Our World in } \\
\text { Data }\end{array}$ & Singapore & $\begin{array}{l}\text { Our World in } \\
\text { Data: C19 Policy } \\
\text { responses }\end{array}$ & COVID-evidence & $\begin{array}{l}\text { Our World in } \\
\text { Data: COVID- } \\
19 \text { vaccinations }\end{array}$ \\
\hline Global 5050 & UK & $\begin{array}{l}\text { IFPRI COVID-19 } \\
\text { Policy Response } \\
\text { Portal }\end{array}$ & Cochrane & \\
\hline $\begin{array}{l}\text { CEBM, } \\
\text { University of } \\
\text { Oxford }\end{array}$ & US & $\begin{array}{l}\text { COVID-19 } \\
\text { Primer }\end{array}$ & Clinicaltrials.gov & \\
\hline $\begin{array}{l}\text { Humanitarian } \\
\text { Data Exchange }\end{array}$ & & NIH LitCovid & UKCDR & \\
\hline $\begin{array}{l}\text { Information is } \\
\text { Beautiful }\end{array}$ & & $\begin{array}{l}\text { WHO COVID-19 } \\
\text { Database }\end{array}$ & & \\
\hline LSHTM & & & & \\
\hline $\begin{array}{l}\text { HealthMap } \\
\text { (cases) }\end{array}$ & & & & \\
\hline $\begin{array}{l}\text { The Commons } \\
\text { Project }\end{array}$ & & & & \\
\hline SeroTracker & & & & \\
\hline
\end{tabular}




\section{C19 Resource Hubs}

\begin{tabular}{|c|c|c|c|c|c|}
\hline Global & $\begin{array}{l}\text { Regional } \\
\text { \& Country }\end{array}$ & $\begin{array}{l}\text { Academic } \\
\text { journals \& } \\
\text { Publishers }\end{array}$ & $\begin{array}{l}\text { Institutes/Centres/ } \\
\text { Funders/Other }\end{array}$ & Health Topics & $\begin{array}{l}\text { Social } \\
\text { Sciences }\end{array}$ \\
\hline $\begin{array}{l}\text { WHO } \\
\text { COVID-19 } \\
\text { pandemic }\end{array}$ & $\begin{array}{l}\text { Africa } \\
\text { CDC }\end{array}$ & $\begin{array}{l}\text { Annals of } \\
\text { Internal } \\
\text { Medicine }\end{array}$ & LSTM & $\begin{array}{l}\text { Stop TB } \\
\text { Partnership }\end{array}$ & SSHAP \\
\hline $\begin{array}{l}\text { WHO risk } \\
\text { communicati } \\
\text { on }\end{array}$ & $\begin{array}{l}\text { African } \\
\text { Union }\end{array}$ & BMJ & LSHTM & & IDA \\
\hline WHO Q\&A & $\begin{array}{l}\text { Nigeria } \\
\text { CDC }\end{array}$ & $\begin{array}{l}\text { Bulletin of the } \\
\text { WHO }\end{array}$ & $\begin{array}{l}\text { ICL MRC Centre } \\
\text { for Global } \\
\text { Infectious Disease } \\
\text { Analysis }\end{array}$ & $\begin{array}{l}\text { Global } \\
\text { Menstrual } \\
\text { Collective }\end{array}$ & $\begin{array}{l}\text { Disability } \\
\text { and } \\
\text { inclusion }\end{array}$ \\
\hline $\begin{array}{l}\text { WHO Global } \\
\text { research }\end{array}$ & $\begin{array}{l}\text { GeoPoll: } \\
\text { SSA }\end{array}$ & $\begin{array}{l}\text { Cambridge } \\
\text { University } \\
\text { Press }\end{array}$ & ODI & $\begin{array}{l}\text { SLH: } \\
\text { Handwashing } \\
\text { in low } \\
\text { resource } \\
\text { settings }\end{array}$ & $\begin{array}{l}\text { Coregroup } \\
\text { IDDC }\end{array}$ \\
\hline $\begin{array}{l}\text { COVID-19 } \\
\text { Solidarity } \\
\text { Response } \\
\text { Fund }\end{array}$ & $\begin{array}{l}\text { Global } \\
\text { Health } \\
\text { Network } \\
\text { Africa }\end{array}$ & Cell Press & $\begin{array}{l}\text { Johns Hopkins } \\
\text { University }\end{array}$ & $\begin{array}{l}\text { RBM } \\
\text { Partnership }\end{array}$ & $\begin{array}{l}\text { Ethics, } \\
\text { health } \\
\text { systems } \\
\& \\
\text { COVID- } \\
19\end{array}$ \\
\hline UN & $\begin{array}{l}\text { African } \\
\text { Academy } \\
\text { of } \\
\text { Sciences }\end{array}$ & Cochrane & $\begin{array}{l}\text { Center for Global } \\
\text { Development }\end{array}$ & $\begin{array}{l}\text { Epidemic } \\
\text { Preparedness } \\
\text { Innovations }\end{array}$ & $\begin{array}{l}\text { Social } \\
\text { Develop } \\
\text { ment } \\
\text { Direct } \\
\text { C19 blog } \\
\text { series }\end{array}$ \\
\hline UN Women & $\begin{array}{l}\text { Africa } \\
\text { Evidence } \\
\text { Network }\end{array}$ & Elsevier & $\begin{array}{l}\text { CMMID } \\
\text { Repository }\end{array}$ & $\begin{array}{l}\text { Southern } \\
\text { Voice }\end{array}$ & $\begin{array}{l}\text { Covid } \\
\text { Collective }\end{array}$ \\
\hline
\end{tabular}




\begin{tabular}{|c|c|c|c|c|}
\hline & & & & $\begin{array}{l}\text { Research } \\
\text { Platform }\end{array}$ \\
\hline UNOCHA & $\begin{array}{l}\text { OCHA } \\
\text { Southern } \\
\text { and } \\
\text { Eastern } \\
\text { Africa } \\
\text { COVID-19 } \\
\text { Digest }\end{array}$ & $\begin{array}{l}\text { Health Policy } \\
\text { and Planning }\end{array}$ & $\begin{array}{l}\text { Norwegian } \\
\text { Institute of Public } \\
\text { Health }\end{array}$ & \\
\hline UNHCR & $\begin{array}{l}\text { South } \\
\text { African } \\
\text { Governme } \\
\text { nt }\end{array}$ & JAMA Network & $\begin{array}{l}\text { Oxford Centre for } \\
\text { Evidence-based } \\
\text { Medicine }\end{array}$ & \\
\hline UNICEF & & The Lancet & HEART & \\
\hline UNESCO & & $\begin{array}{l}\text { medRxiv and } \\
\text { bioRxiv } \\
\text { (Preprints) }\end{array}$ & UKRI & \\
\hline UN WFP & & NEJM & Evidence Aid & \\
\hline GOARN & & $\begin{array}{l}\text { Oxford } \\
\text { University } \\
\text { Press }\end{array}$ & $\mathrm{NIH}$ & \\
\hline EPI-WIN & & PLoS & $\begin{array}{l}\text { IFPRI Resources } \\
\text { and Analyses of } \\
\text { C19 Impact }\end{array}$ & \\
\hline World Bank & & SAGE journals & $\begin{array}{l}\text { Prevent } \\
\text { Epidemics }\end{array}$ & \\
\hline $\begin{array}{l}\text { Our World in } \\
\text { Data }\end{array}$ & & Science & & \\
\hline $\begin{array}{l}\text { COVID-19 } \\
\text { Narratives by } \\
\text { David } \\
\text { Nabarro }\end{array}$ & & Springer Nature & & \\
\hline
\end{tabular}




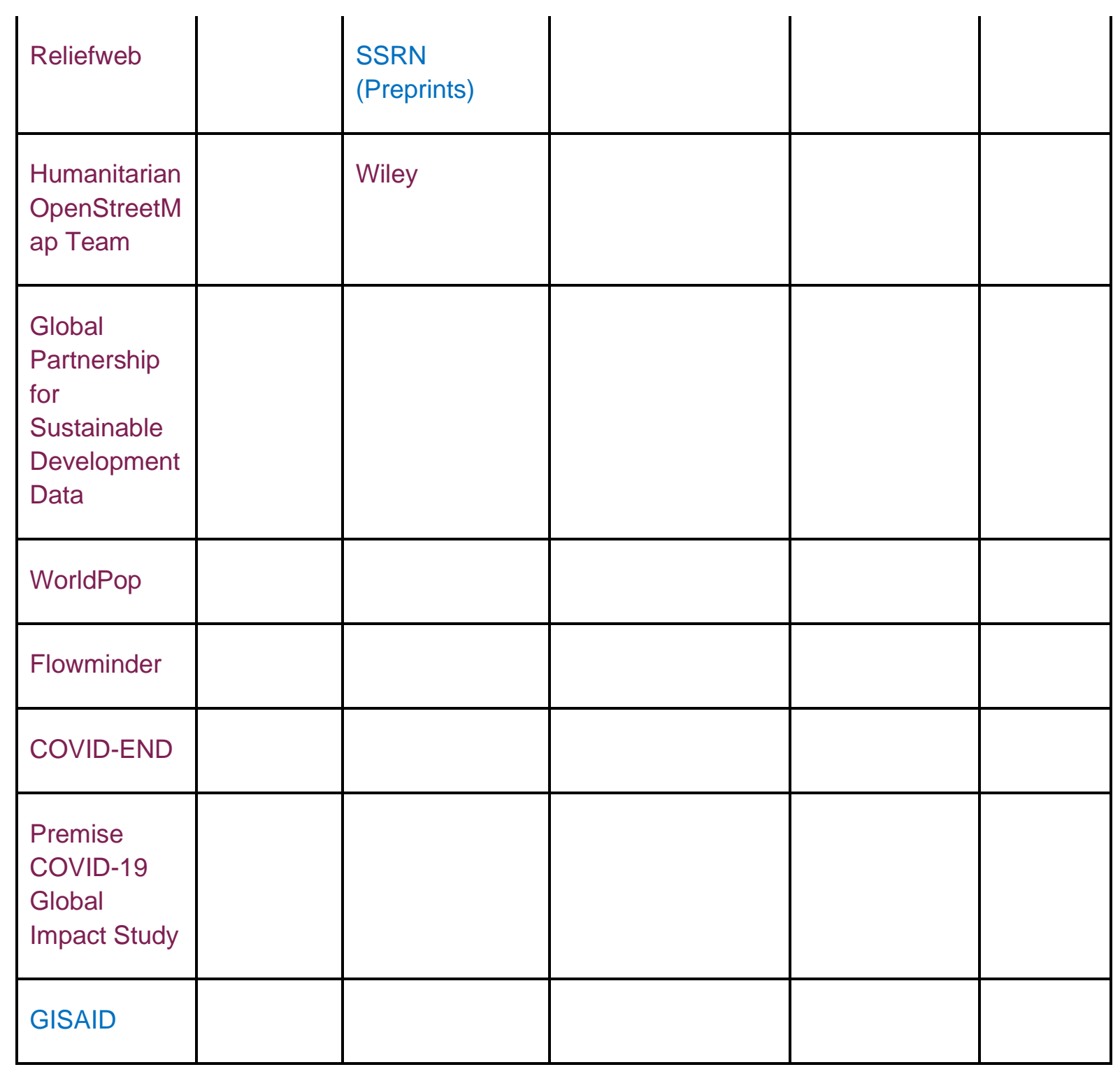




\section{Online learning \& events}

\begin{tabular}{|c|c|c|c|c|}
\hline Date & Title/URL & $\begin{array}{l}\text { Online } \\
\text { learning/event }\end{array}$ & Duration & Lead \\
\hline $\begin{array}{l}18 \text { March } \\
2021\end{array}$ & $\begin{array}{l}\text { Africa taking charge of } \\
\text { its future: prioritizing } \\
\text { gender equality in the } \\
\text { path to recovery }\end{array}$ & Webinar & 1h 30 & CGD \\
\hline $\begin{array}{l}10 \text { March } \\
2021\end{array}$ & $\begin{array}{l}\text { Equity and scale in } \\
\text { global immunization: } \\
\text { new evidence from } \\
\text { Nigeria on cash } \\
\text { transfers for vaccination }\end{array}$ & Webinar & 1h 15 & CGD \\
\hline $\begin{array}{l}9 \text { March } \\
2021\end{array}$ & $\begin{array}{l}\text { COVID-19 vaccines and } \\
\text { Africa: where do we } \\
\text { stand in the race for } \\
\text { vaccines? }\end{array}$ & $\begin{array}{l}\text { Virtual } \\
\text { conference } \\
\text { webinar }\end{array}$ & $1 \mathrm{~h}$ & AHAIC \\
\hline $\begin{array}{l}8 \text { March } \\
2021\end{array}$ & $\begin{array}{l}\text { Chronic Respiratory } \\
\text { Diseases in the COVID } \\
\text { era }\end{array}$ & Webinar & & GARD, WHO \\
\hline $\begin{array}{l}\text { February } \\
2021\end{array}$ & $\begin{array}{l}\text { COVID-19 vaccination } \\
\text { training for health } \\
\text { workers }\end{array}$ & Online training & $3 h$ & WHO \\
\hline 14.01 .2021 & $\begin{array}{l}\text { Evidence to impact in } \\
\text { crisis: how have we } \\
\text { measured up during the } \\
\text { COVID-19 pandemic? }\end{array}$ & Webinar & $1 \mathrm{~h} 30$ & CGD \\
\hline 04.12 .2020 & $\begin{array}{l}\text { COVID-19, supply chain } \\
\text { resilience and global } \\
\text { trade }\end{array}$ & Webinar & $1 \mathrm{~h}$ & CGD \\
\hline 03.12 .2020 & $\begin{array}{l}\text { More money for health } \\
\text { services: What is the } \\
\text { tole of PFM in the "new } \\
\text { normal"? }\end{array}$ & $\begin{array}{l}\text { WHO \& CGD } \\
\text { Health systems } \\
\text { Governance \& } \\
\text { Financing }\end{array}$ & 1h 30 & Joe Kutzin \\
\hline
\end{tabular}




\begin{tabular}{|c|c|c|c|c|}
\hline 01.12 .2020 & $\begin{array}{l}\text { Solutions and support } \\
\text { for the mental wellbeing } \\
\text { of community health } \\
\text { workers on the COVID- } \\
19 \text { frontline }\end{array}$ & Webinar & & $\begin{array}{l}\text { HSG TWG on CHWs } \\
\text { with The George } \\
\text { Institute for Global } \\
\text { Health }\end{array}$ \\
\hline 19.11 .2020 & $\begin{array}{l}\text { Looking at the pandemic } \\
\text { with a gender lens }\end{array}$ & $\begin{array}{l}\text { Live Twitter } \\
\text { conversation }\end{array}$ & & SSHAP \\
\hline 16.11 .2020 & $\begin{array}{l}\text { HIFA and WHO } \\
\text { collaborate to promote } \\
\text { sharing of experience } \\
\text { and expertise around } \\
\text { the maintenance of } \\
\text { essential health services } \\
\text { during (and after) the } \\
\text { pandemic }\end{array}$ & $\begin{array}{l}\text { 4-week } \\
\text { discussion } \\
\text { starting } 16 \text { Nov }\end{array}$ & & HIFA \\
\hline 10.11 .2020 & $\begin{array}{l}\text { COVID-19 vaccine } \\
\text { predictions part } 2: \\
\text { estimating the time } \\
\text { before we approve } \\
\text { efficacious COVID-19 } \\
\text { vaccines }\end{array}$ & Online event & $1 \mathrm{~h} 30$ & CGD \\
\hline 16.10 .2020 & $\begin{array}{l}\text { Financing a Global } \\
\text { Public Health Response }\end{array}$ & Online event & $1 \mathrm{~h} 30$ & CGD \\
\hline 02.10 .2020 & $\begin{array}{l}\text { Understanding and } \\
\text { Improving COVID-19 } \\
\text { Vaccine Portfolio }\end{array}$ & Online event & $1 \mathrm{~h} 30$ & CGD \\
\hline 21.09 .2020 & $\begin{array}{l}\text { Mitigating the Economic } \\
\text { and Health Impact of } \\
\text { COVID-19 across Africa }\end{array}$ & Online event & $1 \mathrm{~h} 30$ & CGD, GF, AU \\
\hline June 2020 & $\begin{array}{l}\text { OpenWHO, the free, } \\
\text { open-access learning } \\
\text { platform for health } \\
\text { emergencies, now offers } \\
10 \text { online courses } \\
\text { related to COVID19. }\end{array}$ & Online courses & Varies & WHO \\
\hline
\end{tabular}




\begin{tabular}{|c|c|c|c|c|}
\hline $\begin{array}{l}\text { Available } \\
\text { now }\end{array}$ & $\begin{array}{l}\text { Standard precautions: } \\
\text { Environmental cleaning } \\
\text { and disinfection }\end{array}$ & Online course & 1 hour & WHO \\
\hline $\begin{array}{l}\text { Available } \\
\text { now }\end{array}$ & $\begin{array}{l}\text { COVID-19: Effective } \\
\text { Nursing in Times of } \\
\text { Crisis }\end{array}$ & Online course & $\begin{array}{l}2 \text { weeks } \\
-2 \\
\text { hours } \\
\text { per } \\
\text { week }\end{array}$ & $\begin{array}{l}\text { Johns Hopkins } \\
\text { School of Nursing }\end{array}$ \\
\hline $\begin{array}{l}\text { Available } \\
\text { now }\end{array}$ & $\begin{array}{l}\text { WHO Academy and } \\
\text { WHO Info mobile } \\
\text { applications }\end{array}$ & Mobile app & & WHO \\
\hline $\begin{array}{l}\text { Available } \\
\text { now }\end{array}$ & $\begin{array}{l}\text { COVID-19: Pandemics, } \\
\text { Modelling and Policy }\end{array}$ & Online learning & $\begin{array}{l}2 \text { weeks } \\
\mid 2 \text { hours } \\
\text { weekly } \\
\text { study }\end{array}$ & $\begin{array}{l}\text { FutureLearn } \\
\text { UNESCO UNITWIN } \\
\text { Complex Systems } \\
\text { Digital Campus/Open } \\
\text { University }\end{array}$ \\
\hline 11.5 .2020 & $\begin{array}{l}\text { COVID-19 Contact } \\
\text { Tracing course }\end{array}$ & Online learning & 5 hours & $\begin{array}{l}\text { Johns Hopkins } \\
\text { Bloomberg School of } \\
\text { Health }\end{array}$ \\
\hline $\begin{array}{l}7-28 \text { May } \\
2020\end{array}$ & Virtual Evidence Weeks & 5 sessions & 1h 30 & $\begin{array}{l}\text { International Initiative } \\
\text { for Impact Evaluation } \\
\text { (3ie) }\end{array}$ \\
\hline $\begin{array}{l}\text { Tuesdays } \\
\text { at } 1700 \\
\text { CEST } \\
\text { (Geneva } \\
\text { time) \& } \\
\text { Thursdays } \\
\text { 0830 CEST } \\
\text { (Geneva } \\
\text { time) }\end{array}$ & $\begin{array}{l}\text { COVID-19 Open online } \\
\text { brief with Dr David } \\
\text { Nabarro }\end{array}$ & Event & $1 \mathrm{~h}$ & $4 S D$ \\
\hline $\begin{array}{l}\text { Available } \\
\text { now }\end{array}$ & $\begin{array}{l}\text { Emerging respiratory } \\
\text { viruses, including } \\
\text { COVID-19: methods for } \\
\text { detection, prevention, } \\
\text { response and control }\end{array}$ & Online learning & 3 hours & WHO \\
\hline
\end{tabular}




\begin{tabular}{|c|c|c|c|c|}
\hline $\begin{array}{l}\text { Available } \\
\text { now }\end{array}$ & $\begin{array}{l}\text { Responding to COVID- } \\
\text { 19: Real-time training } \\
\text { for the coronavirus } \\
\text { disease outbreak }\end{array}$ & Online learning & $\begin{array}{l}\text { Multiple } \\
\text { self- } \\
\text { paced } \\
\text { course }\end{array}$ & WHO \\
\hline $\begin{array}{l}25 \text { May } \\
2020\end{array}$ & $\begin{array}{l}\text { COVID-19: Tackling the } \\
\text { Novel Coronavirus }\end{array}$ & Online learning & $\begin{array}{l}3 \text { weeks } \\
\text { | } 4 \text { hours } \\
\text { weekly } \\
\text { study }\end{array}$ & $\begin{array}{l}\text { FutureLearn } \\
\text { LSHTM/UK PHRST }\end{array}$ \\
\hline $\begin{array}{l}\text { Available } \\
\text { online now } \\
\text { without } \\
\text { mentors. } \\
\text { Updated } \\
\text { version will } \\
\text { commence } \\
\text { early June } \\
2020\end{array}$ & $\begin{array}{l}\text { COVID-19 Diagnostics } \\
\text { and Testing }\end{array}$ & Online learning & $\begin{array}{l}3 \text { weeks } \\
\text { | } 3 \text { hours } \\
\text { weekly } \\
\text { study }\end{array}$ & $\begin{array}{l}\text { FutureLearn } \\
\text { FIND/LSHTM/ASLM }\end{array}$ \\
\hline $\begin{array}{l}6 \text { April } \\
2020\end{array}$ & $\begin{array}{l}\text { COVID-19 Critical Care: } \\
\text { Understanding and } \\
\text { Application }\end{array}$ & Online learning & $\begin{array}{l}5 \text { weeks } \\
\text { | } 1 \text { hour } \\
\text { weekly } \\
\text { study }\end{array}$ & $\begin{array}{l}\text { FutureLearn } \\
\text { University of } \\
\text { Edinburgh \& Royal } \\
\text { College of Physicians } \\
\text { of Edinburgh }\end{array}$ \\
\hline $\begin{array}{l}\text { Available } \\
\text { now }\end{array}$ & $\begin{array}{l}\text { COVID-19 supporting } \\
\text { online courses }\end{array}$ & Online learning & $\begin{array}{l}\text { Multiple } \\
\text { self- } \\
\text { paced } \\
\text { course }\end{array}$ & BMJ Learning \\
\hline
\end{tabular}

\section{Suggested citation}

Millington, K.A. (2021). COVID-19 Health Evidence Summary No.116. K4D Evidence Summary.

Brighton, UK: Institute of Development Studies. DOI: 10.19088/K4D.2021.035 


\section{Rapid review methodology}

The rapid weekly search for peer-reviewed literature is carried out through a PubMed search with the following keywords ("COVID-19" OR "severe acute respiratory syndrome coronavirus 2" OR "2019-nCoV" OR "SARS-CoV2" OR "2019nCoV" OR "coronavirus" ) AND ("Africa" OR "South Asia" OR "Developing" OR "low-income" OR "low income" OR "lower-middle income" OR "low and middle income" OR "LMIC" OR "LIC" OR "global south") OR ("poverty") OR ("equity" OR "equities"), restricted to articles published in the previous 2 to 3 days, in English. This is complemented by a search of the homepage of the following high-impact global health journals: The Lancet journals, New England Journal of Medicine, Nature, JAMA, Annals of Internal Medicine, Cochrane Reviews, BMJ Global Health, the PLoS journals and a Twitter search of their Twitter pages. A search also of preprints from bioRxiv and medRxiv. Please note that papers that have not been peer-reviewed are highlighted in red. All primary research papers that relate to the primary and secondary impacts of the COVID-19 response in LMICs, and disease control and health system responses are included. Articles related to tackling the secondary impacts on other sectors are not included. Additional commentaries, opinions, and commissioned pieces are selected based on relevance.

The search for dashboards, guidelines, tools, editorials, comments, blogs, opinions and news is through the academic journals listed above, $\mathrm{C} 19$ resource hubs and following lead academics and professionals on Twitter.

\section{About this report}

This weekly COVID-19 health evidence summary (HES) is based on 3.5 hours of desk-based research. The summary is not intended to be a comprehensive summary of available evidence on COVID-19 but aims to make original documents easily accessible to decision makers which, if relevant to them, they should go to before making decisions. The HES are not intended to replace medical or professional advice and the researcher or the K4D consortium cannot be held responsible for any decisions made about COVID-19 on the basis of the HES alone. K4D services are provided by a consortium of leading organisations working in international development, led by the Institute of Development Studies (IDS), with Education Development Trust, Itad, University of Leeds Nuffield Centre for International Health and Development, Liverpool School of Tropical Medicine (LSTM), University of Birmingham International Development Department (IDD) and the University of Manchester Humanitarian and Conflict Response Institute (HCRI).

This evidence summary was prepared for the UK Government's Foreign, Commonwealth and Development Office (FCDO) and its partners in support of pro-poor programmes. Except where otherwise stated, it is licensed for non-commercial purposes under the terms of the Open Government Licence v3.0. K4D cannot be held responsible for errors, omissions or any consequences arising from the use of information contained in this health evidence summary. Any views and opinions expressed do not necessarily reflect those of FCDO, K4D or any other contributing organisation.

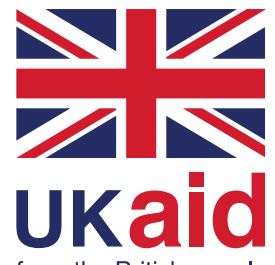

(C) Crown copyright 2021 